\title{
A simple scoring system to estimate perioperative mortality following liver resection for primary liver malignancy-the Hepatectomy Risk Score (HeRS)
}

\author{
Dimitrios Moris", Brian I. Shaw", Cecilia Ong, Ashton Connor, Mariya L. Samoylova, Samuel J. Kesseli, \\ Nader Abraham, Jared Gloria, Robin Schmitz, Zachary W. Fitch, Bryan M. Clary, Andrew S. Barbas \\ Department of Surgery, Duke University Medical Center, Durham, NC, USA \\ Contributions: (I) Conception and design: D Moris, BI Shaw, C Ong, A Connor, BM Clary, AS Barbas; (II) Administrative support: None; (III) \\ Provision of study materials or patients: None; (IV) Collection and assembly of data: None; (V) Data analysis and interpretation: All authors; (VI) \\ Manuscript writing: All authors; (VII) Final approval of manuscript: All authors. \\ \#These authors contributed equally to this work. \\ Correspondence to: Dimitrios Moris MD, MSc, PhD, DUMC. Department of Surgery, Duke University Medical Center, Durham, NC 27710, USA. \\ Email: dimitrios.moris@duke.edu.
}

Background: Selection of the optimal treatment modality for primary liver cancers remains complex, balancing patient condition, liver function, and extent of disease. In individuals with preserved liver function, liver resection remains the primary approach for treatment with curative intent but may be associated with significant mortality. The purpose of this study was to establish a simple scoring system based on Model for End-stage Liver Disease (MELD) and extent of resection to guide risk assessment for liver resections.

Methods: The 2005-2015 NSQIP database was queried for patients undergoing liver resection for primary liver malignancy. We first developed a model that incorporated the extent of resection (1 point for major hepatectomy) and a MELD-Na score category of low (MELD-Na =6, 1 point), medium (MELD-Na =7-10, 2 points) or high (MELD-Na $>10,3$ points) with a score range of $1-4$, called the Hepatic Resection Risk Score (HeRS). We tested the predictive value of this model on the dataset using logistic regression. We next developed an optimal multivariable model using backwards sequential selection of variables under logistic regression. We performed K-fold cross validation on both models. Receiver operating characteristics were plotted and the optimal sensitivity and specificity for each model were calculated to obtain positive and negative predictive values.

Results: A total of 4,510 patients were included. HeRS was associated with increased odds of 30-day mortality [HeRS =2: OR =3.23 (1.16-8.99), $\mathrm{P}=0.025$; HeRS =3: OR =6.54 (2.39-17.90), $\mathrm{P}<0.001$; HeRS =4: $\mathrm{OR}=13.69$ (4.90-38.22), $\mathrm{P}<0.001]$. The AUC for this model was 0.66. The AUC for the optimal multivariable model was higher at 0.76 . Under K-fold cross validation, the positive predictive value (PPV) and negative predictive value (NPV) of these two models were similar at PPV $=6.4 \%$ and NPV $=97.7 \%$ for the HeRS only model and PPV $=8.4 \%$ and NPV $=98.1 \%$ for the optimal multivariable model.

Conclusions: The HeRS offers a simple heuristic for estimating 30-day mortality after resection of primary liver malignancy. More complicated models offer better performance but at the expense of being more difficult to integrate into clinical practice.

Keywords: Hepatocellular carcinoma (HCC); cholangiocarcinoma; liver resection; Model for End-stage Liver Disease (MELD); outcomes

Submitted Sep 10, 2019. Accepted for publication Oct 28, 2019.

doi: $10.21037 / \mathrm{hbsn} .2020 .03 .12$

View this article at: http://dx.doi.org/10.21037/hbsn.2020.03.12 


\section{Introduction}

Primary liver cancer is currently the second most common cause of cancer-related death worldwide $(1,2)$. It demonstrates the greatest increase in both mortality and morbidity in the United States during the past 2 decades $(3,4)$. Primary liver cancer comprises a heterogeneous group of malignant tumors with different histological features and prognosis that range from hepatocellular carcinoma (HCC) and intrahepatic cholangiocarcinoma (iCCA) to mixed hepatocellular cholangiocarcinoma (HCC-CCA), fibrolamellar HCC (FLC), and the pediatric neoplasm hepatoblastoma (5).

HCC accounts for over $70 \%$ of primary liver malignancies with over 750,000 new cases and nearly equal number of cancer-related deaths each year $(6,7)$. Surgery, in the form of either liver resection or transplantation, remains the mainstay of treatment for patients with resectable HCC (8-10). Intrahepatic cholangiocarcinoma (iCCA) is the second most common primary liver malignancy and its incidence and mortality are increasing worldwide and in the US (11-13). Surgical resection is the mainstay for treatment of resectable iCCA (14) with liver transplantation remaining controversial (15).

Safety of elective hepatectomies for primary liver malignancies in patients with or without cirrhosis has increased significantly during the last decades but mortality of such procedures is still estimated between $3 \%$ and $14 \%(16,17)$. A key determinant of "resectability" is the anticipated volume of the future liver remnant; for patients without hepatic dysfunction, a remnant volume of $>25 \%$ or $>250 \mathrm{~mL} / \mathrm{m}^{2}$ is typically recommended $(18,19)$. However, patients with pre-existing liver disease have less hepatic reserve, and prediction of postoperative liver decompensation remains difficult (20). Post-hepatectomy liver failure is the most severe complication, with a reported mortality as high as $50 \%(21,22)$. Moreover, it is the leading cause of prolonged hospitalization, increased costs, and poor longterm outcomes in patients undergoing surgical procedure.

The Model for End-stage Liver Disease (MELD) has been shown to reflect hepatocellular reserve and mortality risk in patients with cirrhosis and has been used to stratify end-stage liver disease patients for liver transplantation organ allocation $(23,24)$. In patients with cirrhosis, the preoperative MELD score has been shown to be a strong predictor of both perioperative mortality and long-term survival for patients undergoing hepatic resection for HCC $(21,25)$.

In the current era of multidisciplinary patient care, selection of the optimal treatment modality for primary liver cancers remains complex, balancing patient condition, liver function, and extent of disease. To date, several systems have been proposed to inform prognosis and guide treatment decisions for patients with HCC (26-29). Of interest, there are only a few prognostic tools available for patients with ICC $(30,31)$. In addition, most of these tools rely on factors known only after surgery as only a few models have utilized exclusively preoperative factors to stratify early and late postoperative outcomes $(31,32)$.

The purpose of this study was to establish a simple scoring system based on MELD sodium (MELD-Na), which is currently used for organ allocation in liver transplantation, and extent of resection to guide risk assessment for liver resection of primary liver malignancies. We present the following article in accordance with the STARD reporting checklist (available at https://hbsn. amegroups.com/article/view/10.21037/hbsn.2020.03.12/rc).

\section{Methods}

\section{Ethical Statement}

The study was conducted in accordance with the Declaration of Helsinki (as revised in 2013). The study was approved by institutional review board (IRB\#Pro00103324) and informed consent was taken from individual participants where applicable.

\section{Cobort definition}

Data from the National Surgical Quality Improvement Project (NSQIP) were extracted for the years 2005-2015. Patients with a post-operative diagnosis of code 155 .* ("Malignant neoplasm of liver and intrahepatic bile ducts"), who underwent one of four well defined procedures (CPT 47120-hepatectomy, resection of liver; partial lobectomy, CPT 47125-hepatectomy, resection of liver, total left lobectomy; CPT 47122-hepatectomy, resection of the liver, trisegmentectomy; and 47130-hepatectomy, resection of the liver, total right lobectomy) were included in our analysis. Patients who were missing outcome data, missing lab values needed to calculate the MELD-Na in the 30 days prior to surgery (INR, Total Bilirubin, Creatinine, and Sodium), who underwent emergency surgery, or were missing variables needed for our multivariable analyses were excluded.

Of note, a substantial portion of patients in this cohort were coded with a diagnosis code of 155.1 , which is 


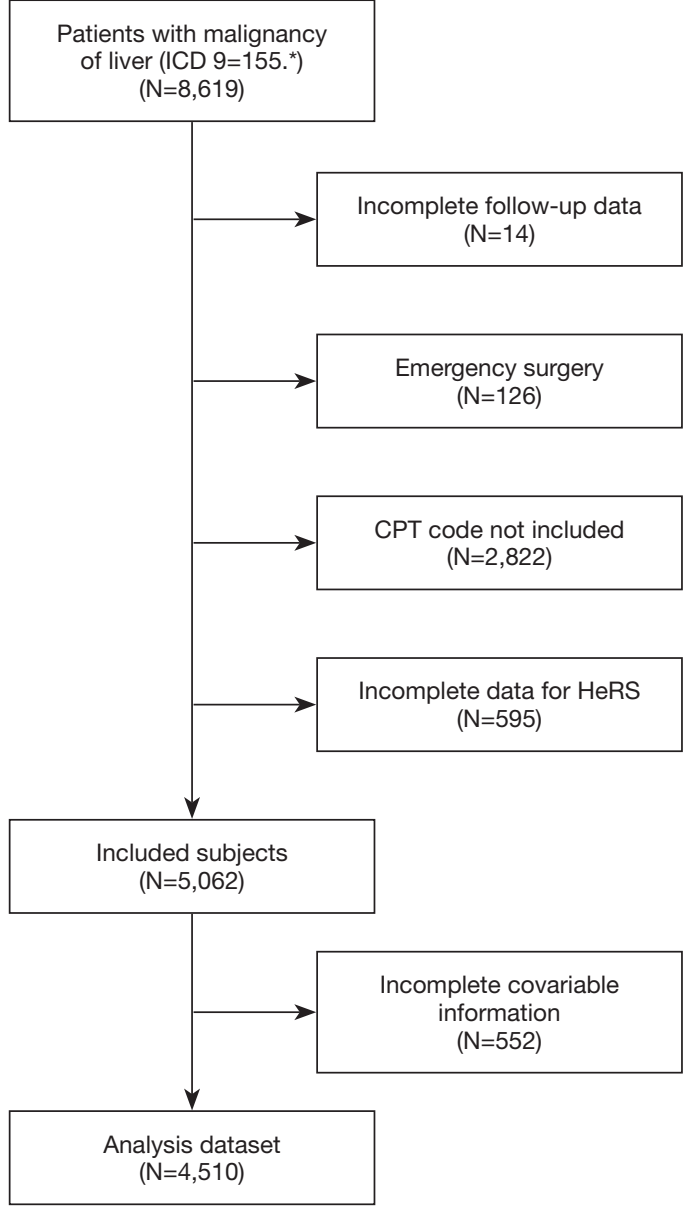

Figure 1 Patient flow chart. Patients were initially identified by a post-operative diagnosis of liver cancer as defined by ICD-9 code 155. All 155 subcodes were included. Patients were excluded if they did not undergo one of four known hepatectomy codes $(\mathrm{CPT}=47120,4712,47122$, and 47130). Patients were also excluded if they did not have data for any one of the following variables: age, sex, BMI, ASA class $>2$, functional status other than independent, smoker within 1 year of surgery, dyspnea (on exertion or at rest), ascites at the time of surgery, weight loss $>10 \%$ in last 6 months, platelet count $<100$, albumin at time of surgery, history of COPD, history of HTN, and history of insulin dependent diabetes. CPT, current procedural terminology.

specifically "Malignant neoplasm of the intrahepatic bile ducts" (n=984, 21\%; Table S1). Because cholangiocarcinoma is thought to be different than HCC, a sensitivity analysis for all below analyses using only those patients with a diagnosis of 155.0 ("Malignant neoplasm of the liver, primary"), which is essentially HCC only, was also completed.

\section{Development of HeRS}

The HeRS was defined a priori to include MELD-Na and the extent of hepatic resection. Hepatic resection was considered "major" if the patient underwent a procedure associated with CPT codes 47122 (trisegmentectomy) or 47130 (right hepatectomy); "minor" hepatectomies were defined by CPT codes 47120 (partial hepatectomy) or 47125 (left hepatectomy). The MELD-Na was defined a priori as low if it was 6 (no evidence of disease). Patients with abnormal MELD-Na scores (7 or greater) were stratified into two groups for optimal sensitivity and specificity for mortality using the Youden index (33). A cutoff of 10 was determined using this method. A patient was assigned a score of 1 or 0 for a major or a minor hepatectomy, respectively, and a score of 1 (MELD-Na =6), 2 (MELD-Na 7-10), or 3 (MELD-Na >10) for their MELD-Na category. The HeRS was calculated by first determining the patients MELD-Na categorization $(1,2$, or 3$)$ and then adding one if the patient had undergone a major hepatectomy. The minimum value for the HeRS was defined as 1 and the maximum 4.

\section{Statistical analysis}

Descriptive statistics were summarized for the cohort. 30day mortality was compared among HeRS groups using chi-squared. Simple logistic regression and Firth logistic regression for 30-day mortality was performed with HeRS as the predictor variable and receiver operating characteristics (ROC) curves plotted. In order to interrogate what residual risk was not captured by the HeRS, other presumed predictors of perioperative mortality were included in a multivariable logistic regression model with the HeRS. Sequential stepwise backwards selection with a cutoff of $\mathrm{P}<0.05$ was performed to find the optimal model which included the HeRS. K-fold cross validation was used to avoid overfitting when determining receiver operating characteristics of both the univariate model with HeRS alone and the optimal multivariable model. The Youden index was again used to define the cutoff for maximum sensitivity and specificity of this model to determine positive- and negativepredictive value (PPV and NPV). All statistical analyses were performed in STATA 15 (College Station, TX).

\section{Results}

\section{Cobort definition and demographics}

A total of 8,619 patients were identified in the NSQIP 
Table 1 Patient demographics and characteristics

\begin{tabular}{|c|c|c|c|c|c|}
\hline Variables & $\mathrm{HeRS}=1$ & $\mathrm{HeRS}=2$ & $\mathrm{HeRS}=3$ & $\mathrm{HeRS}=4$ & Total cohort \\
\hline Total, n [\%] & $476[10]$ & $1,877[42]$ & $1,676[37]$ & $481[11]$ & 4,510 \\
\hline Sex, female, n [\%] & $268[56]$ & $744[40]$ & $568[34]$ & $151[31]$ & $1731[38]$ \\
\hline \multicolumn{6}{|l|}{ Race, n [\%] } \\
\hline Asian & $44[9]$ & $183[10]$ & $136[8]$ & 38 [8] & $401[9]$ \\
\hline Black or African American & $40[8]$ & $189[10]$ & $160[9]$ & $35[8]$ & 424 [9] \\
\hline Native Hawaiian or Pacific Islander & $1[1]$ & 9 [1] & $3[1]$ & $3[1]$ & $16[1]$ \\
\hline Unknown & $41[9]$ & $138[7]$ & 146 [9] & $52[11]$ & $377[8]$ \\
\hline BMI, median [Q1-Q3] & 27 [24-31] & 27 [24-31] & 27 [24-31] & 26 [23-30] & 27 [24-31] \\
\hline ASA class $\geq 3, n[\%]$ & $348[73]$ & $1,474[79]$ & $1,365[81]$ & $405[82]$ & $3,592[80]$ \\
\hline Dependent functional status, $\mathrm{n}$ [\%] & $1[1]$ & $24[1]$ & $21[1]$ & 7 [1] & $53[1]$ \\
\hline Smoker, w/in 1 year, n [\%] & $102[21]$ & $410[22]$ & 367 [22] & $88[18]$ & $967[21]$ \\
\hline Dyspnea on exertion or at rest, $\mathrm{n}$ [\%] & $41[9]$ & $153[8]$ & $153[9]$ & $33[7]$ & $380[8]$ \\
\hline Ascites, n [\%] & $0[0]$ & $32[2]$ & $43[3]$ & $19[4]$ & $94[2]$ \\
\hline History of COPD, n [\%] & $25[5]$ & $105[6]$ & $96[6]$ & $24[5]$ & $250[6]$ \\
\hline Hypertension, n [\%] & $237[50]$ & $1072[57]$ & 1018 [61] & $295[61]$ & $2622[58]$ \\
\hline Insulin dependent diabetes, $\mathrm{n}$ [\%] & $22[5]$ & 139 [7] & $188[11]$ & $48[10]$ & 397 [9] \\
\hline Bleeding disorder history, $\mathrm{n}$ [\%] & $13[3]$ & $77[4]$ & $86[5]$ & $28[6]$ & 204 [5] \\
\hline \multicolumn{6}{|l|}{ Operation type, n [\%] } \\
\hline Minor hepatectomy/lobectomy & $476[100]^{\#}$ & $1,646[88]$ & $778[46]$ & $0[0]$ & $2,900[64]$ \\
\hline Major hepatectomy & $0[0]$ & $231[12]$ & $898[51]$ & $481[100]^{\&}$ & $1610[36]$ \\
\hline MELD-Na, median [Q1-Q3] & $6^{*}$ & $8[7-9]$ & $10[8-13]$ & 13 [12-16] & 9 [7-11] \\
\hline Mortality, n [\%] & $4[1]$ & $50[3]$ & 88 [5] & $50[10]$ & $192[4]$ \\
\hline
\end{tabular}

*, by definition, MELD-Na must be 6 for HRRS to equal 1; ", by definition, all HRRS 1 had to have a minor hepatectomy; ${ }^{\text {, }}$, by definition, all HRRS 4 had to have a major hepatectomy. BMI, basic metabolic index; COPD, chronic obstructive pulmonary disease; HeRS, hepatectomy risk score; MELD-NA, Model for End-stage Liver Disease- sodium; ASA, American society of Anesthesiologists. 


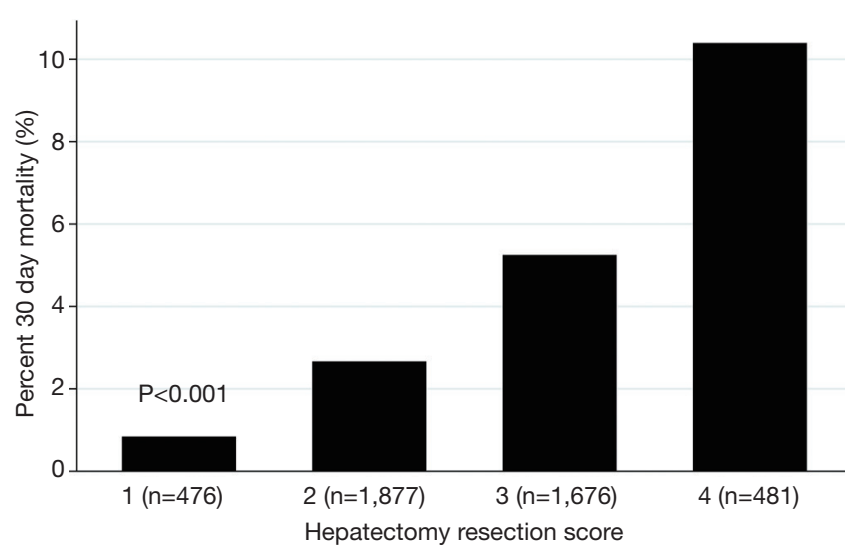

Figure 2 The 30-day mortality by HeRS. The 30-day mortality for each HeRS is plotted as bars. The distribution of mortality was significantly varied by $\mathrm{Chi}^{2}$ test $(\mathrm{P}<0.001)$. The absolute number of patients in each group is shown below each bar. HeRS, hepatectomy risk score.

Table 2 HeRS only logistic regression model

\begin{tabular}{lcc}
\hline Variable & OR estimate $(95 \% \mathrm{Cl})$ & P value \\
\hline Intercept & $0.008(0.003-0.023)$ & $<0.001$ \\
HeRS $=1$ & 1.0 (reference) & N/A \\
HeRS =2 & $3.23(1.16-8.99)$ & 0.025 \\
HeRS =3 & $6.54(2.39-17.9)$ & $<0.001$ \\
HeRS =4 & $13.69(4.90-382)$ & $<0.001$ \\
\hline
\end{tabular}

OR estimates for the HeRS only model. HeRS, hepatectomy risk score; Cl, confidence interval; N/A, not applicable; OR, odds ratio. database between 2005 and 2015 who met inclusion criteria. After exclusions, our total study cohort was 4,510 (Figure 1). Patient demographics and characteristics are summarized in Table 1. Overall, the cohort was elderly, with a median age of 64 (Q1-Q3: 56-71), mostly male (72\%), and mostly white $(67 \%)$. The median MELD-Na was 9 (Q1-Q3: 7-11) and most patients underwent a minor hepatectomy (64\%). The postoperative 30-day mortality varied significantly by HeRS as demonstrated in Figure 2.

\section{Mortality prediction using HeRS alone}

To determine how well the HeRS score predicts postoperative mortality, we performed a logistic regression on mortality using the HeRS as the predictor. Given that the HeRS yields 4 categories, we obtained 3 odds ratios (with HeRS score of 1 as the reference category) for HeRS $=2[\mathrm{OR}=3.23$ (1.16-8.99), $\mathrm{P}=0.025], \mathrm{HeRS}=3[\mathrm{OR}=6.54$ (2.39-17.9), $\mathrm{P}<0.001]$, and $\mathrm{HeRS}=4$ [OR $=13.69$ (4.9038.22), $\mathrm{P}<0.001$ ] (Table 2). ROC analysis yielded an AUC of 0.66 (Figure $3 A$ ). To ensure the analysis was not biased due to the relatively low incidence of mortality, we performed Firth Logistic Regression, which yielded a similar OR for each HeRS level (Table S2).

\section{Multivariable model for mortality prediction}

In order to investigate the performance of a model utilizing the HeRS alone to a more comprehensive model, multivariable logistic regression analysis was performed
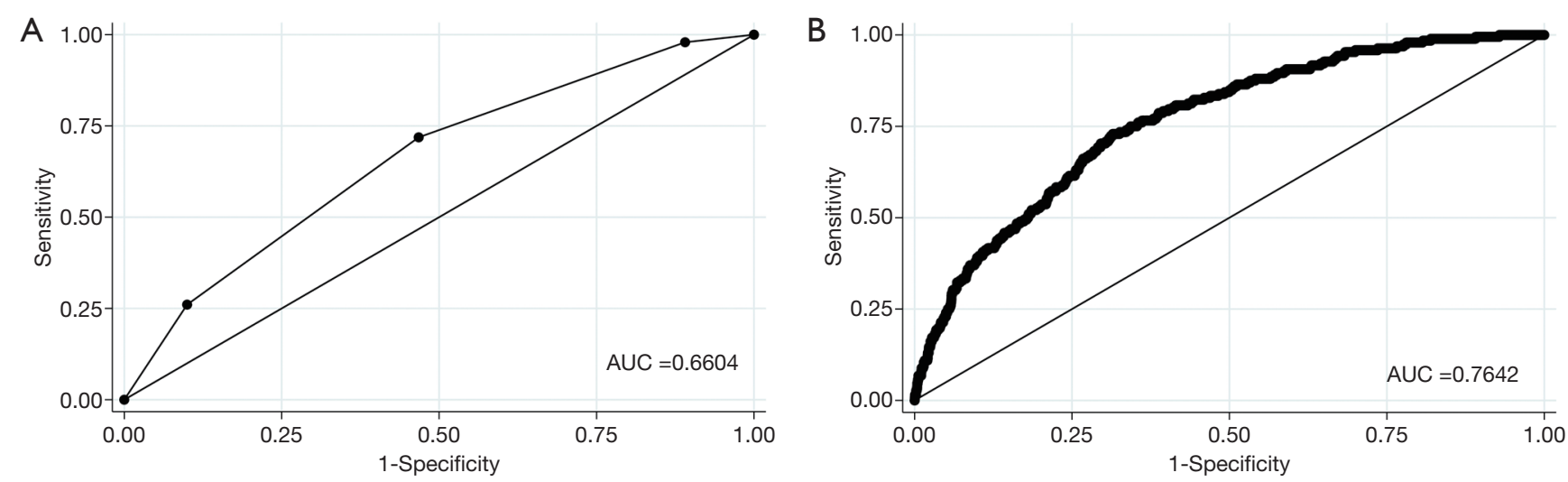

Figure 3 (A) HeRS only ROC curve; (B) ROC curve for optimal multivariable model. HeRS, hepatectomy risk score; ROC, receiver operating characteristics; AUC, area under the curve. 
including the following variables: age, sex, body mass index (BMI), American Society of Anesthesiologists (ASA) class, functional status, smoking status, dyspnea (on exertion or at rest), presence of ascites, weight loss $>10 \%$ in last 6 months, platelet count $<100$, albumin at time of surgery, steroid use at time of surgery, history of chronic obstructive pulmonary

Table 3 Optimal multivariable model

\begin{tabular}{|c|c|c|}
\hline Variable & OR estimate $(95 \% \mathrm{Cl})$ & $P$ value \\
\hline Intercept & $0.006(0.001-0.036)$ & $<0.001$ \\
\hline $\mathrm{HeRS}=1$ & 1.0 (reference) & N/A \\
\hline $\mathrm{HeRS}=2$ & $2.30(0.82-6.46)$ & 0.113 \\
\hline $\mathrm{HeRS}=3$ & $3.87(1.40-10.73)$ & $<0.009$ \\
\hline $\mathrm{HeRS}=4$ & $7.25(2.55-20.65)$ & $<0.001$ \\
\hline Age (years) & $1.03(1.02-1.05)$ & $<0.001$ \\
\hline Sex $(1=$ male, $0=$ female $)$ & $1.50(1.07-2.10)$ & 0.018 \\
\hline $\begin{array}{l}\text { ASA class }(1=\text { greater } \\
\text { than } 2 ; 0=\text { less than } 2)\end{array}$ & $2.38(1.33-4.24)$ & 0.003 \\
\hline $\begin{array}{l}\text { Functional status } \\
\text { ( } 1=\text { dependent, } \\
0=\text { independent })\end{array}$ & $2.91(1.33-6.38)$ & 0.008 \\
\hline $\begin{array}{l}\text { Platelet count }<100 \\
(1=\text { yes, } 0=\text { no })\end{array}$ & $3.32(2.14-5.16)$ & $<0.001$ \\
\hline $\begin{array}{l}\text { Albumin at time of } \\
\text { surgery }(\mathrm{g} / \mathrm{dL})\end{array}$ & $0.53(0.41-0.68)$ & $<0.001$ \\
\hline
\end{tabular}

disease (COPD), history of hypertension, history of insulin dependent diabetes, and history of a bleeding disorder. Description of the full multivariable model and ROC curve can be found in Table S3 and Figure S1. Sequential backwards selection eventually yielded an optimal formula with the variables of age, sex, ASA class, functional status, platelets $<100$, and pre-operative albumin as well as the HeRS. Of note, though one level of the HeRS was not statistically significantly different than the reference value (2 points $v s .1$ point) it was included in the final model for completeness (Table 3). A ROC curve was generated with an AUC of 0.76 (Figure 3B).

\section{K-fold cross validation}

In order to ensure that the model was not overfit, we reperformed the analysis using $\mathrm{k}$-fold cross validation with 5 groups. Under the HeRS only condition, the AUC was 0.64 . Under the optimal multivariable logistic regression, the AUC was 0.75 . The PPV for the HeRS only model was $6.4 \%$ and the NPV was $97.7 \%$. For the optimal multivariable model, the PPV was $8.4 \%$ and NPV was $98.2 \%$ (Table 4).

\section{Sensitivity analysis with HCC only}

Given concerns that intrahepatic cholangiocarcinoma is biologically differently than HCC, we compared mortality at a given HeRS between the two groups. There were no significant differences in mortality between patients with HCC versus those with intrahepatic cholangiocarcinoma

Table 4 K-Fold Cross Validation for HeRS-only model, full multivariable model, and optimal multivariable model

\begin{tabular}{|c|c|c|c|}
\hline Formula & AUC & PPV (\%) & NPV (\%) \\
\hline HeRS only & 0.64 & 6.4 & 97.7 \\
\hline Full multivariable & 0.74 & 8.3 & 97.9 \\
\hline Optimal multivariable & 0.75 & 8.4 & 98.2 \\
\hline HeRS only & 0.62 & 5.5 & 97.7 \\
\hline Full multivariable & 0.71 & 6.4 & 97.8 \\
\hline Optimal multivariable & 0.74 & 7.7 & 98.1 \\
\hline
\end{tabular}

AUC, PPV and NPV for test sets after $n=5 \mathrm{~K}$-fold cross validation. PPV and NPV were optimized by the Youden Index for the test set. All values are reported for the test set. AUC, area under the curve; PPV, positive predictive value; NPV, negative predictive value; HeRS, hepatectomy risk score. 
(Figure S2). However, we still performed a sensitivity analysis where we repeated our analysis on only those patients with HCC. This is summarized in Tables S4-S6 and Figure S3. Finally, we performed K-fold cross validation on this subset of patients and obtained the results presented in Table 4. Ultimately, the estimates were similar with an AUC of 0.62 for the HeRS only and 0.74 for the optimal multivariable model.

\section{Discussion}

Preoperative assessment of liver function and prediction of postoperative functional reserve are of paramount importance to minimize surgical risk of liver resection (34). In the present study, we propose a simple model, the HeRS that is significantly correlated with 30-day mortality and has fair test characteristics in spite of its simplicity. Though a more complex multivariable model performs better in our analysis, the relative infrequency of mortality within 30 days leads to similar PPV and NPV for the HeRS and more complex multivariate model. Therefore, we argue that the simplicity of the HeRS is a benefit, not a detriment, and can serve as a useful heuristic for determining the risk for early postoperative mortality. In practice, clinicians continue to use the Child-Turcotte-Pugh (CTP) score to determine eligibility for hepatic resection in spite of a lack of validation (35). This suggests that simpler scoring systems have intrinsic properties that are useful to practicing clinicians, such as the HeRS introduced here.

It was recently shown that for patients with HCC and a MELD score $>10$, liver transplantation offered superior survival. This benefit was eliminated in cases of major vascular involvement or in patients with MELD scores $<10$ (36). Specifically, patients with a MELD score of $\geq 10$ had a $47 \% 5$-year survival after resection, compared to patients with a MELD score less than 10 having a 5-year survival of $67 \%(\mathrm{P}<0.0001)$. Mean 5 -year LT benefit was -4.50 months ( $95 \%$ CI: -4.73 to -4.27 ) for patients with a MELD score of $<10$, and 0.81 months (95\% CI 0.58 to 1.04) for those with a MELD score of $\geq 10$ (36). Similarly, a recent study demonstrated that a MELD score $>7.24$ can be an important predictor of post-hepatectomy mortality or metastasis and may prompt a detailed assessment with the provided risk calculator (37).

To date, there are multiple models predicting 30-day mortality after HCC resection (38-41). Also, there are several studies comparing MELD score alone to novel scoring systems as predictors of outcomes after liver resection in patients with HCC. A large Korean study evaluated platelet-albumin-bilirubin (PALBI) and albumin-bilirubin (ALBI) versus CTP class and MELD score to predict overall survival. The PALBI grade had a higher AUC than the CTP class, MELD score, or ALBI grade (overall AUC: 0.675 vs. 0.633, 0.645, and 0.642, respectively; $\mathrm{P}<0.001$ ) (42). In the same setting, Mai et al. showed that preoperative aspartate aminotransferaseto-platelet-ratio index (APRI) score predicted posthepatectomy liver failure significantly more accurate than Child-Pugh, MELD, or ALBI scores. The optimal sensitivity and specificity of the APRI score for predicting post-hepatectomy liver failure were $72.2 \%$ and $68.0 \%$, respectively (43). In the setting of iCCA there are no previous studies using MELD score as predictor of early postoperative mortality after resection. Tsilimigras et al. recently showed that higher ALBI grade was related with prolonged length-of-stay, increased perioperative transfusion needs and higher early mortality after liver resection for iCCA (44). Also Zhang et al. demonstrated that major complications and early postoperative death were more common after a major versus minor hepatic resection (both $\mathrm{P}<0.01)(14)$.

There are some strengths and limitations of this study that deserve mention. The primary strength of the analysis is the large sample size derived from a large cohort of centers in the United States, which likely makes these results generalizable in different practice settings. A significant limitation of the NSQIP database, however, is the lack of more detailed clinical information, such as degree of cirrhosis, extent of operative resection, and postoperative pathologic analysis. We have only analyzed the performance of the HeRS in one dataset and therefore it would benefit from external validation in a separate dataset. Additionally, the primary outcome of postoperative mortality within 30 days is relatively rare, which makes logistic regression subject to bias; however, we addressed this by including analysis with Firth Logistic regression, which accounts for small event counts (45).

In conclusion, we present a simple and reliable score to assist with risk assessment in patients being considered for liver resection for primary liver cancer. Though the HeRS does not achieve the same sensitivity and specificity as some contemporary multivariable models, it is intuitive, simple to calculate, and performs favorably when compared to multiple previously published models. Ease of integration into clinical practice should be considered when developing predictive models. 


\section{Acknowledgments}

Funding: None.

\section{Footnote}

Reporting Checklist: The authors have completed the STARD reporting checklist. Available at https://hbsn.amegroups. com/article/view/10.21037/hbsn.2020.03.12/rc

Conflicts of Interest: All authors have completed the ICMJE uniform disclosure form (available at https://hbsn. amegroups.com/article/view/10.21037/hbsn.2020.03.12/coif). The authors have no conflicts of interest to declare.

Ethical Statement: The authors are accountable for all aspects of the work in ensuring that questions related to the accuracy or integrity of any part of the work are appropriately investigated and resolved. The study was conducted in accordance with the Declaration of Helsinki (as revised in 2013). The study was approved by institutional review board (IRB\#Pro00103324) and informed consent was taken from individual participants where applicable.

Open Access Statement: This is an Open Access article distributed in accordance with the Creative Commons Attribution-NonCommercial-NoDerivs 4.0 International License (CC BY-NC-ND 4.0), which permits the noncommercial replication and distribution of the article with the strict proviso that no changes or edits are made and the original work is properly cited (including links to both the formal publication through the relevant DOI and the license). See: https://creativecommons.org/licenses/by-nc-nd/4.0/.

\section{References}

1. Murray CJ, Vos T, Lozano R, et al. Disability-adjusted life years (DALYs) for 291 diseases and injuries in 21 regions, 1990-2010: a systematic analysis for the Global Burden of Disease Study 2010. Lancet 2012;380:2197-223.

2. Argyrou C, Moris D, Vernadakis S. Hepatocellular carcinoma development in non-alcoholic fatty liver disease and non-alcoholic steatohepatitis. Is it going to be the "Plague" of the 21st century? A literature review focusing on pathogenesis, prevention and treatment. J BUON 2017;22:6-20.

3. Torre LA, Bray F, Siegel RL, et al. Global cancer statistics, 2012. CA Cancer J Clin 2015;65:87-108.
4. Njei B, Rotman Y, Ditah I, et al. Emerging trends in hepatocellular carcinoma incidence and mortality. Hepatology 2015;61:191-9.

5. Flejou JF. WHO Classification of digestive tumors: the fourth edition. Ann Pathol 2011;31:S27-31.

6. Beal EW, Tumin D, Kabir A, et al. Trends in the Mortality of Hepatocellular Carcinoma in the United States. J Gastrointest Surg 2017;21:2033-8.

7. Lafaro KJ, Demirjian AN, Pawlik TM. Epidemiology of hepatocellular carcinoma. Surg Oncol Clin N Am 2015;24:1-17.

8. European Association for the Study of the Liver. EASL Clinical Practice Guidelines: Management of hepatocellular carcinoma. J Hepatol 2018;69:182-236.

9. Marrero JA, Kulik LM, Sirlin CB, et al. Diagnosis, Staging, and Management of Hepatocellular Carcinoma: 2018 Practice Guidance by the American Association for the Study of Liver Diseases. Hepatology 2018;68:723-50.

10. Tsilimigras DI, Bagante F, Moris D, et al. Defining the chance of cure after resection for hepatocellular carcinoma within and beyond the Barcelona Clinic Liver Cancer guidelines: A multi-institutional analysis of 1,010 patients. Surgery 2019;166:967-74.

11. Patel T. Increasing incidence and mortality of primary intrahepatic cholangiocarcinoma in the United States. Hepatology 2001;33:1353-7.

12. Beal EW, Tumin D, Moris D, et al. Cohort contributions to trends in the incidence and mortality of intrahepatic cholangiocarcinoma. Hepatobiliary Surg Nutr 2018;7:270-6.

13. Global Burden of Disease Cancer Collaboration; Fitzmaurice C, Allen C, et al. Global, Regional, and National Cancer Incidence, Mortality, Years of Life Lost, Years Lived With Disability, and Disability-Adjusted Lifeyears for 32 Cancer Groups, 1990 to 2015: A Systematic Analysis for the Global Burden of Disease Study. JAMA Oncol 2017;3:524-48.

14. Zhang XF, Bagante F, Chakedis J, et al. Perioperative and Long-Term Outcome for Intrahepatic Cholangiocarcinoma: Impact of Major Versus Minor Hepatectomy. J Gastrointest Surg 2017;21:1841-50.

15. Rana A, Hong JC. Orthotopic liver transplantation in combination with neoadjuvant therapy: a new paradigm in the treatment of unresectable intrahepatic cholangiocarcinoma. Curr Opin Gastroenterol 2012;28:258-65.

16. Berzigotti A, Reig M, Abraldes JG, et al. Portal hypertension and the outcome of surgery for hepatocellular 
carcinoma in compensated cirrhosis: a systematic review and meta-analysis. Hepatology 2015;61:526-36.

17. Tsilimigras DI, Mehta R, Moris D, et al. Utilizing Machine Learning for Pre- and Postoperative Assessment of Patients Undergoing Resection for BCLC-0, A and B Hepatocellular Carcinoma: Implications for Resection Beyond the BCLC Guidelines. Ann Surg Oncol 2020;27:866-74.

18. Thirunavukarasu P, Aloia TA. Preoperative Assessment and Optimization of the Future Liver Remnant. Surg Clin North Am 2016;96:197-205.

19. Kubota K, Makuuchi M, Kusaka K, et al. Measurement of liver volume and hepatic functional reserve as a guide to decision-making in resectional surgery for hepatic tumors. Hepatology 1997;26:1176-81.

20. Moris D, Vernadakis S, Papalampros A, et al. The effect of Guidelines in surgical decision making: The paradigm of hepatocellular carcinoma. J BUON 2016;21:1332-6.

21. Citterio D, Facciorusso A, Sposito C, et al. Hierarchic Interaction of Factors Associated With Liver Decompensation After Resection for Hepatocellular Carcinoma. JAMA Surg 2016;151:846-53.

22. Moris D, Tsilimigras DI, Kostakis ID, et al. Anatomic versus non-anatomic resection for hepatocellular carcinoma: A systematic review and meta-analysis. Eur J Surg Oncol 2018;44:927-38.

23. Kamath PS, Wiesner RH, Malinchoc M, et al. A model to predict survival in patients with end-stage liver disease. Hepatology 2001;33:464-70.

24. Wiesner RH, McDiarmid SV, Kamath PS, et al. MELD and PELD: application of survival models to liver allocation. Liver Transpl 2001;7:567-80.

25. Teh SH, Christein J, Donohue J, et al. Hepatic resection of hepatocellular carcinoma in patients with cirrhosis: Model of End-Stage Liver Disease (MELD) score predicts perioperative mortality. J Gastrointest Surg 2005;9:120715; discussion 15.

26. Xu Q, Yan Y, Gu S, et al. A Novel InflammationBased Prognostic Score: The Fibrinogen/Albumin Ratio Predicts Prognoses of Patients after Curative Resection for Hepatocellular Carcinoma. J Immunol Res 2018;2018:4925498.

27. Wang YY, Zhong JH, Su ZY, et al. Albumin-bilirubin versus Child-Pugh score as a predictor of outcome after liver resection for hepatocellular carcinoma. Br J Surg 2016;103:725-34.

28. Hsu HY, Yu MC, Lee CW, et al. RAM score is an effective predictor for early mortality and recurrence after hepatectomy for hepatocellular carcinoma. BMC Cancer 2017;17:742.

29. Delis SG, Bakoyiannis A, Dervenis C, et al. Perioperative risk assessment for hepatocellular carcinoma by using the MELD score. J Gastrointest Surg 2009;13:2268-75.

30. Wang Y, Li J, Xia Y, et al. Prognostic nomogram for intrahepatic cholangiocarcinoma after partial hepatectomy. J Clin Oncol 2013;31:1188-95.

31. Buettner S, Galjart B, van Vugt JLA, et al. Performance of prognostic scores and staging systems in predicting long-term survival outcomes after surgery for intrahepatic cholangiocarcinoma. J Surg Oncol 2017;116:1085-95.

32. Hyder O, Marques H, Pulitano C, et al. A nomogram to predict long-term survival after resection for intrahepatic cholangiocarcinoma: an Eastern and Western experience. JAMA Surg 2014;149:432-8.

33. Fluss R, Faraggi D, Reiser B. Estimation of the Youden Index and its associated cutoff point. Biom J 2005;47:458-72.

34. Dimitroulis D, Damaskos C, Valsami S, et al. From diagnosis to treatment of hepatocellular carcinoma: An epidemic problem for both developed and developing world. World J Gastroenterol 2017;23:5282-94.

35. Abbas N, Makker J, Abbas H, et al. Perioperative Care of Patients With Liver Cirrhosis: A Review. Health Serv Insights 2017;10:1178632917691270.

36. Vitale A, Huo TL, Cucchetti A, et al. Survival Benefit of Liver Transplantation Versus Resection for Hepatocellular Carcinoma: Impact of MELD Score. Ann Surg Oncol 2015;22:1901-7.

37. Fromer MW, Aloia TA, Gaughan JP, et al. The utility of the MELD score in predicting mortality following liver resection for metastasis. Eur J Surg Oncol 2016;42:1568-75.

38. Sahara K, Paredes AZ, Merath K, et al. Evaluation of the ACS NSQIP Surgical Risk Calculator in Elderly Patients Undergoing Hepatectomy for Hepatocellular Carcinoma. J Gastrointest Surg 2020;24:551-9.

39. Takagi K, Umeda Y, Yoshida R, et al. Preoperative Controlling Nutritional Status Score Predicts Mortality after Hepatectomy for Hepatocellular Carcinoma. Dig Surg 2019;36:226-32.

40. Shen J, Tang L, Zhang X, et al. A Novel Index in Hepatocellular Carcinoma Patients After Curative Hepatectomy: Albumin to Gamma-Glutamyltransferase Ratio (AGR). Front Oncol 2019;9:817.

41. Tokumitsu Y, Shindo Y, Matsui H, et al. Utility of scoring systems combining the product of tumor number and size 
with liver function for predicting the prognosis of patients with hepatocellular carcinoma after hepatectomy. Oncol Lett 2019;18:3903-13.

42. Lee SK, Song MJ, Kim SH, et al. Comparing various scoring system for predicting overall survival according to treatment modalities in hepatocellular carcinoma focused on Platelet-albumin-bilirubin (PALBI) and albuminbilirubin (ALBI) grade: A nationwide cohort study. PLoS One 2019;14:e0216173.

43. Mai RY, Ye JZ, Long ZR, et al. Preoperative aspartate aminotransferase-to-platelet-ratio index as a predictor of

Cite this article as: Moris D, Shaw BI, Ong C, Connor A, Samoylova ML, Kesseli SJ, Abraham N, Gloria J, Schmitz R, Fitch ZW, Clary BM, Barbas AS. A simple scoring system to estimate perioperative mortality following liver resection for primary liver malignancy-the Hepatectomy Risk Score (HeRS). HepatoBiliary Surg Nutr 2021;10(3):315-324. doi: 10.21037/hbsn.2020.03.12 posthepatectomy liver failure for resectable hepatocellular carcinoma. Cancer Manag Res 2019;11:1401-14.

44. Tsilimigras DI, Hyer JM, Moris D, et al. Prognostic utility of albumin-bilirubin grade for short- and longterm outcomes following hepatic resection for intrahepatic cholangiocarcinoma: A multi-institutional analysis of 706 patients. J Surg Oncol 2019;120:206-13.

45. Greenland S, Mansournia MA. Penalization, bias reduction, and default priors in logistic and related categorical and survival regressions. Stat Med 2015;34:3133-43. 
Table S1 Distribution of cases by post-operative diagnosis

\begin{tabular}{lccccc}
\hline Post-op diagnosis & HeRS $=1$ & HeRS $=2$ & HeRS $=3$ & HeRS $=4$ & Total cohort \\
\hline Total, $\mathrm{n}[\%]$ & $476[11]$ & $1,877[42]$ & $1,676[37]$ & $481[10]$ & 4,510 \\
$155.0, \mathrm{n}[\%]$ & $313[66]$ & $1,360[72]$ & $1,134[67]$ & $274[57]$ & $3,081[68]$ \\
$155.1, \mathrm{n}[\%]$ & $96[20]$ & $312[17]$ & $382[23]$ & $166[34]$ & $956[21]$ \\
$155.2, \mathrm{n}[\%]$ & $40[8]$ & $132[7]$ & $97[6]$ & $26[5]$ & $295[7]$ \\
155 (unspecified), $\mathrm{n}[\%]$ & $27[6]$ & $73[4]$ & $63[4]$ & $15[3]$ & $178[4]$ \\
\hline
\end{tabular}

Diagnosis codes are specified as follows: 155.0, malignant neoplasm of liver, primary; 155.1, malignant neoplasm of intrahepatic bile ducts; 155.2, malignant neoplasm of liver, not specified as primary or secondary. HeRS, hepatectomy risk score.

Table S2 Firth logistic regression for HeRS only model

\begin{tabular}{lcc}
\hline Variable & OR estimate $(95 \% \mathrm{Cl})$ & $\mathrm{P}$ value \\
\hline Intercept & $0.009(0.004-0.024)$ & $<0.001$ \\
$\mathrm{HeRS}=1$ & 1.0 (reference) & $\mathrm{N} / \mathrm{A}$ \\
$\mathrm{HeRS}=2$ & $2.90(1.10-7.65)$ & 0.031 \\
$\mathrm{HeRS}=3$ & $5.85(2.25-15.16)$ & $<0.001$ \\
$\mathrm{HeRS}=4$ & $12.29(4.64-32.51)$ & $<0.001$ \\
\hline
\end{tabular}

HeRS, hepatectomy risk score; OR, odds ratio; Cl, confidence interval; N/A, not applicable.

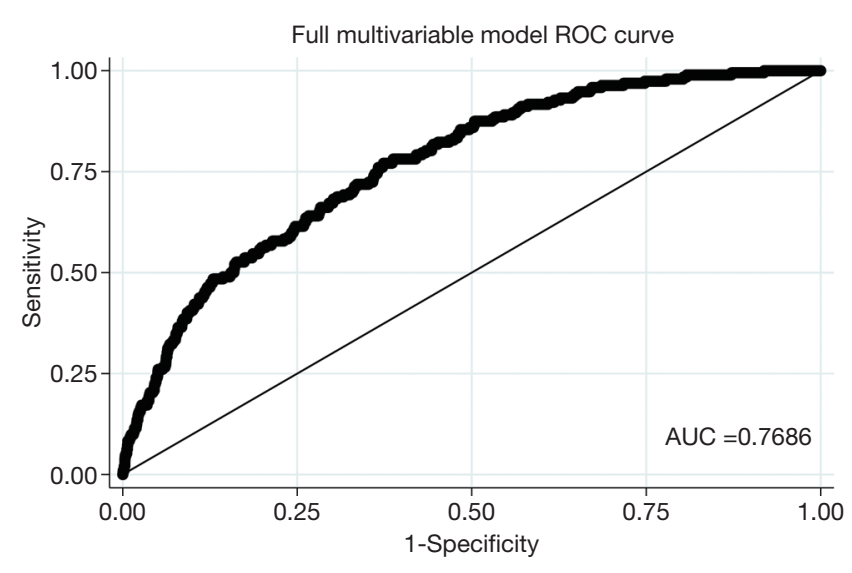

Figure S1 Full multivariable model ROC curve.

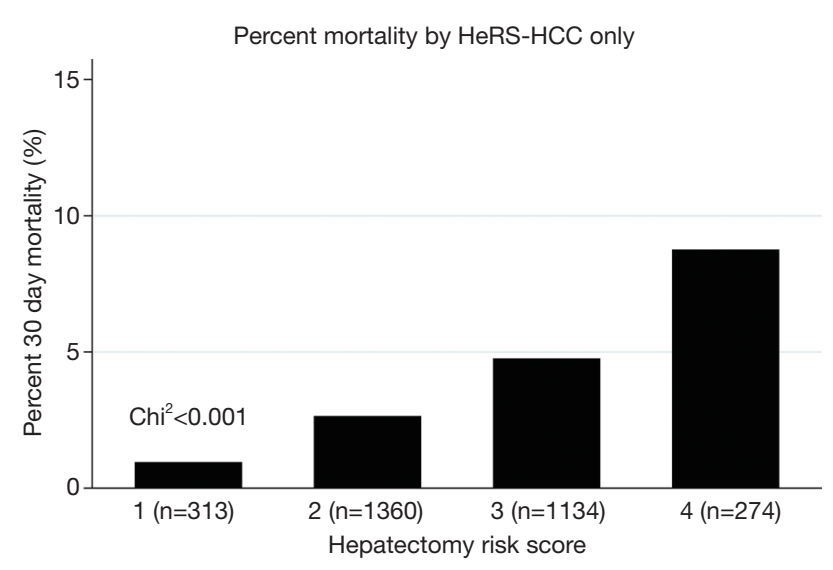

Figure S2 Mortality by HeRS for primary liver parenchymal and biliary ductal intrahepatic neoplasms.

Table S3 Full multivariable logistic regression

\begin{tabular}{|c|c|c|}
\hline Variable & OR estimate $(95 \% \mathrm{Cl})$ & $P$ value \\
\hline Intercept & $0.003(0.0004-0.021)$ & $<0.001$ \\
\hline $\mathrm{HeRS}=1$ & 1.0 (reference) & N/A \\
\hline $\mathrm{HeRS}=2$ & $2.35(0.84-6.60)$ & 0.104 \\
\hline $\mathrm{HeRS}=3$ & $3.93(1.42-10.90)$ & $<0.009$ \\
\hline $\mathrm{HeRS}=4$ & $7.51(2.63-21.44)$ & $<0.001$ \\
\hline Age (in years) & $1.03(1.02-1.05)$ & $<0.001$ \\
\hline Sex $(1=$ male, $0=$ female $)$ & $1.55(1.10-2.18)$ & 0.012 \\
\hline BMI & $1.02(0.99-1.05)$ & 0.150 \\
\hline ASA class ( $1=$ greater than $2 ; 0=$ less than 2$)$ & $2.27(1.26-4.07)$ & 0.006 \\
\hline Functional status ( $1=$ dependent, $0=$ independent) & $2.88(1.30-6.37)$ & 0.009 \\
\hline Smoker within 1 year of surgery $(1=$ yes, $0=$ no) & $1.18(0.80-1.72)$ & 0.407 \\
\hline Dyspnea at rest or on exertion ( $1=$ yes, $0=$ no) & $1.08(0.66-1.78)$ & 0.747 \\
\hline Ascites at time of surgery $(1=$ yes, $0=$ no) & $0.613(0.25-1.51)$ & 0.284 \\
\hline Weight Loss $>10 \%$ in past 6 months $(1=$ yes, $0=$ no) & $1.43(0.85-2.40)$ & 0.177 \\
\hline Platelet count $<100$ ( $1=$ yes, $0=$ no) & $3.58(2.26-5.66)$ & $<0.001$ \\
\hline Albumin at time of surgery (g/dL) & $0.53(0.41-0.69)$ & $<0.001$ \\
\hline Steroid use at time of surgery ( $1=$ yes, $0=$ no) & $2.02(1.02-4.02)$ & 0.045 \\
\hline History of COPD ( $1=$ yes, $0=$ no) & $0.93(0.50-1.74)$ & 0.823 \\
\hline History of hypertension ( $1=$ yes, $0=$ no) & $0.91(0.64-1.28)$ & 0.575 \\
\hline History of insulin dependent diabetes $(1=$ yes, $0=$ no) & $1.14(0.73-1.80)$ & 0.560 \\
\hline History of bleeding disorder & $0.93(0.51-1.70)$ & 0.811 \\
\hline
\end{tabular}

Odds ratio (OR) estimates for the Full Multivariable Model. HeRS, hepatectomy risk score; $\mathrm{Cl}$, confidence interval; ASA, American Society of Anesthesiologists; COPD, chronic obstructive pulmonary disease; BMI, basic metabolic index; N/A, not applicable. 
Table S4 HeRS only logistic regression-neoplasm of primary liver parenchyma only analysis ( $\mathrm{n}=3,081)$

\begin{tabular}{lcc}
\hline Variable & OR estimate $(95 \% \mathrm{Cl})$ & P value \\
\hline Intercept & $0.01(0.003-0.03)$ & $<0.001$ \\
$\mathrm{HeRS}=1$ & 1.0 (reference) & $\mathrm{N} / \mathrm{A}$ \\
$\mathrm{HeRS}=2$ & $2.8(0.86-9.18)$ & 0.087 \\
$\mathrm{HeRS}=3$ & $5.17(1.60-16.63)$ & 0.006 \\
$\mathrm{HeRS}=4$ & $9.92(2.95-33.32)$ & $<0.001$ \\
\hline
\end{tabular}

OR, odds ratio; HeRS, hepatectomy risk score; $\mathrm{Cl}$, confidence interval; N/A, not applicable.

Table S5 Full multivariable model for neoplasm of primary liver parenchyma only analysis

\begin{tabular}{|c|c|c|}
\hline Variable & OR estimate $(95 \% \mathrm{Cl})$ & $P$ value \\
\hline Intercept & $0.008(0.006-0.10)$ & $<0.001$ \\
\hline $\mathrm{HeRS}=1$ & 1.0 (reference) & $\mathrm{N} / \mathrm{A}$ \\
\hline $\mathrm{HeRS}=2$ & $1.90(0.57-6.30)$ & 0.293 \\
\hline $\mathrm{HeRS}=3$ & $2.66(0.81-8.75)$ & 0.11 \\
\hline $\mathrm{HeRS}=4$ & $4.5(1.31-15.8)$ & 0.02 \\
\hline Age (in years) & $1.03(1.01-1.06)$ & 0.002 \\
\hline Sex $(1=$ male, $0=$ female $)$ & $1.72(1.08-2.76)$ & 0.023 \\
\hline BMI & $1.01(0.98-1.05)$ & 0.400 \\
\hline ASA class $(1=$ greater than $2 ; 0=$ less than 2$)$ & $2.12(1.00-4.47)$ & 0.049 \\
\hline Functional status $(1=$ dependent, $0=$ independent $)$ & $2.75(0.98-7.70)$ & 0.054 \\
\hline Smoker within 1 year of surgery $(1=$ yes, $0=$ no $)$ & $1.20(0.75-1.92)$ & 0.447 \\
\hline Dyspnea at rest or on exertion $(1=$ yes, $0=$ no) & $0.96(0.50-1.83)$ & 0.905 \\
\hline Ascites at time of surgery $(1=$ yes, $0=$ no $)$ & $0.40(0.11-1.41)$ & 0.153 \\
\hline Weight loss $>10 \%$ in past 6 months $(1=$ yes, $0=$ no $)$ & $1.32(0.65-2.69)$ & 0.445 \\
\hline Platelet count $<100(1=$ yes, $0=$ no $)$ & $3.53(2.06-6.03)$ & $<0.001$ \\
\hline Albumin at time of surgery $(\mathrm{g} / \mathrm{dL})$ & $0.43(0.31-0.61)$ & $<0.001$ \\
\hline Steroid use at time of surgery $(1=$ yes, $0=$ no) & $1.65(0.63-4.32)$ & 0.304 \\
\hline History of COPD $(1=$ yes, $0=$ no $)$ & $1.01(0.46-2.23)$ & 0.982 \\
\hline History of hypertension $(1=$ yes, $0=$ no) & $0.92(0.59-1.43)$ & 0.710 \\
\hline History of insulin dependent diabetes $(1=$ yes, $0=$ no) & $1.29(0.75-2.21)$ & 0.353 \\
\hline History of bleeding disorder & $0.96(0.47-1.98)$ & 0.922 \\
\hline
\end{tabular}

$\mathrm{OR}$, odds ratio; HeRS, hepatectomy risk score; $\mathrm{Cl}$, confidence interval; ASA, American Society of Anesthesiologists; COPD, chronic obstructive pulmonary disease; BMI, basic metabolic index; N/A, not applicable.

Table S6 Optimal multivariable model for neoplasm of primary liver parenchyma only analysis

\begin{tabular}{lcc}
\hline Variable & OR estimate $(95 \% \mathrm{Cl})$ & $\mathrm{P}$ value \\
\hline Intercept & $0.013(0.001-0.12)$ & $<0.001$ \\
$\mathrm{HeRS}=1$ & 1.0 (reference) & $\mathrm{N} / \mathrm{A}$ \\
$\mathrm{HeRS}=2$ & $1.90(0.58-6.29)$ & 0.291 \\
$\mathrm{HeRS}=3$ & $2.66(0.81-8.745)$ & 0.106 \\
HeRS $=4$ & $4.59(1.33-15.84)$ & 0.016 \\
Age (in years) & $1.03(1.01-1.05)$ & 0.001 \\
Sex $(1=$ male, $0=$ female) & $1.65(1.04-2.63)$ & 0.033 \\
ASA class $(1=$ greater than $2 ; 0=$ less than 2) & $2.26(1.08-4.71)$ & 0.030 \\
Platelet count $<100(1=$ yes, $0=$ no) & $3.25(1.94-5.42)$ & $<0.001$ \\
Albumin at time of surgery $(\mathrm{g} / \mathrm{dL})$ & $0.43(0.31-0.60)$ & $<0.001$ \\
\hline
\end{tabular}

OR, odds ratio; HeRS, hepatectomy risk score; Cl, confidence interval; ASA, American Society of Anesthesiologists; N/A, not applicable.

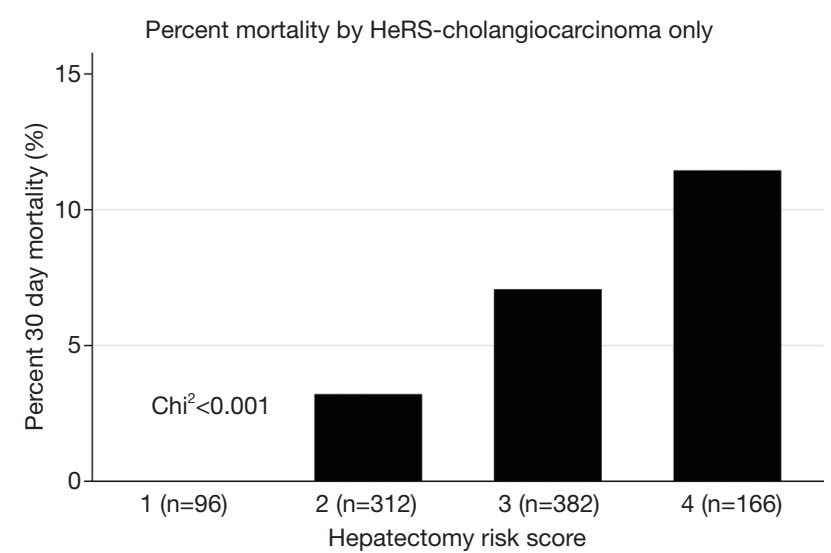

Figure S3 ROC curves for neoplasm of primary liver parenchyma only analysis. 